\title{
PROPERTIES OF SOLUTIONS OF STOCHASTIC DIFFERENTIAL EQUATIONS WITH RANDOM COEFFICIENTS, NON-LIPSCHITZIAN DIFFUSION, AND POISSON MEASURES
}

UDC 519.21

\author{
V. P. ZUBCHENKO
}

\begin{abstract}
The existence and uniqueness of a solution of a stochastic differential equation with random coefficients, non-Lipschitzian diffusion, and with centered as well as with non-centered Poisson measures are proved. We estimate the probability that a solution eventually becomes negative. We find conditions for the existence of a nonnegative solution.
\end{abstract}

\section{INTRODUCTION}

Studies of stochastic diffusion type differential equations with a Hölderian diffusion coefficient are of special interest in view of applications in finance. Equations of this type are widely used for modeling financial markets. In particular, the dynamics of the short term interest rate $r(t)$ in the stochastic Cox-Ingersoll-Ross model [1] is described by the following stochastic differential equation:

$$
d r(t)=k(\gamma-r(t)) d t+\sigma \sqrt{r(t)} d W(t)
$$

where $W$ is a Wiener process and $k, \gamma$, and $\sigma$ are positive constants. In the Cox-IngersollRoss model, $r(t)$ is nonnegative and approaches the constant $\gamma$, which is called the long term interest rate. Of special interest for banks and insurance companies are studies of another long term interest rate, namely

$$
\frac{1}{t} \int_{0}^{t} r(s) d s
$$

where $r(s)$ is the short term interest rate whose behavior is determined, for example, by the stochastic model (11).

However the interest rates in financial markets may have jumps under certain conditions. Thus generalizations of the stochastic Cox-Ingersoll-Ross model are important from both points of view, the practical as well as the theoretical one. The dynamics of the interest rate in these generalizations may be described by a stochastic differential equation with a Hölder diffusion and with Poisson measure [2.

The existence and uniqueness of solutions of stochastic differential equations are considered in a number of papers and monographs. In particular, these problems are considered in 3 and 4 for stochastic differential equations with Lipschitz coefficients and

2010 Mathematics Subject Classification. Primary 60H10; Secondary 60H05, 60J65.

Key words and phrases. Stochastic differential equations, non-Lipschitzian diffusion, Poisson measure, existence and uniqueness of a solution, nonnegativity of a solution, ruin probability.

The author is indebted to the European Commission for their support in the framework of the "Marie Curie Actions" program, grant PIRSES-GA-2008-230804. 
with a jump term as well as for equations with non-Lipschitz diffusion but without a jump term. The existence and uniqueness of solutions of a system of differential equations with respect to a martingale and with a centered Poisson measure and nonrandom non-Lipschitz coefficients is proved in the paper [5]. The existence of a solution of a stochastic differential equation with a linear drift coefficient, with an additional randomness, and with a Hölder diffusion but without a jump term is proved in [6]. The existence and uniqueness of a solution of a stochastic differential equation with bounded coefficients, non-Lipschitzian diffusion, centered or non-centered Poisson measures are considered in [7].

In this paper, we prove a theorem on the existence and uniqueness of the strong solution of a stochastic differential equation with random coefficients, non-Lipschitzian diffusion, and with centered as well as with non-centered Poisson measures. Similarly to [8] (Theorem 1 in $\S 7$ ), [3] (Remark 2 to Theorem 1 in $\S 1$ of Chapter 4), and [9] (Chapter 14) we assume that the conditions imposed on the coefficients of the equation hold uniformly with respect to the space of elementary random events.

The paper is organized as follows. In Section 3. we prove a result on the existence of a nonnegative solution for equations of the type we discussed above. In Section 4 , we obtain an upper bound for the probability that a solution of a stochastic differential equation assumes a negative value. This result is of a practical interest for estimating the ruin probability of an insurance company whose reserve capital is modeled with the help of a stochastic differential equation involving a non-Lipschitzian diffusion and centered Poisson measure.

Let a probability space $(\Omega, \mathcal{F}, \mathrm{P})$ be given and let the flow of $\sigma$-algebras $\mathcal{F}_{t}$ be completed with all random events of $\mathcal{F}_{0}$ of zero probability. We study the following stochastic differential equation:

$$
\begin{aligned}
X(t)= & \zeta(t)+\int_{0}^{t} a(X(s)) d s+\int_{0}^{t} g(X(s)) d W(s) \\
& +\int_{0}^{t} \int_{\mathbb{R}} q_{1}(X(s), y) \tilde{\nu}(d s, d y)+\int_{0}^{t} \int_{\mathbb{R}} q_{2}(X(s), y) \mu(d s, d y),
\end{aligned}
$$

where $\zeta: \Omega \times \mathbb{R}^{+} \rightarrow \mathbb{R}$ is a right continuous stochastic process without discontinuities of the second kind; $W$ a Wiener process; $\nu$ a Poisson measure such that $\mathrm{E} \nu(d t, d y)=$ $\Pi(d y) d t ; \tilde{\nu}(d t, d y)=\nu(d t, d y)-\Pi(d y) d t$ a centered Poisson measure, where $\Pi$ is a $\sigma$ finite measure on the $\sigma$-algebra of Borel sets of $\mathbb{R} ; \mu$ a noncentered Poisson measure such that $\mathrm{E} \mu(d t, d y)=m(d y) d t$, and where $m$ is a finite measure on the $\sigma$-algebra of Borel sets of $\mathbb{R}$. The Wiener process $W$, centered Poisson measure $\tilde{\nu}$, noncentered Poisson measure $\mu$, and stochastic process $\zeta$ are adapted to the flow $\mathcal{F}_{t}$ and are jointly independent. The coefficients $a(x, \omega), g(x, \omega), q_{1}(x, y, \omega)$, and $q_{2}(x, y, \omega)$ are random functions being measurable in all their arguments. The drift coefficient $a(x, \omega)$ is Lipschitzian with probability one, the diffusion $g(x, \omega)$ is Hölderian with probability one (the precise conditions on the diffusion coefficient are stated in the corresponding results below). The definition of the integrals in equation (2) is given, for example, in [3].

\section{Existence AND UNIQUENESS OF THE STRONG SOLUTION}

Theorem 2.1. Let the coefficients of equation (2) be such that

1. $\zeta: \Omega \times \mathbb{R}^{+} \rightarrow \mathbb{R}$ and

$$
\lim _{t_{1} \rightarrow t_{2}} \mathrm{E}\left|\zeta\left(t_{1}\right)-\zeta\left(t_{2}\right)\right|=0
$$


2. for all $x \in \mathbb{R}$,

$$
|a(x, \omega)| \leq l(1+|x|), \quad \int_{\mathbb{R}}\left|q_{1}(x, y, \omega)\right|^{2} \Pi(d y) \leq l
$$

with probability one, where $l$ is a nonrandom constant;

3. with probability one,

$$
\lim _{x_{1} \rightarrow x_{2}} \int_{\mathbb{R}}\left|q_{1}\left(x_{1}, y, \omega\right)-q_{1}\left(x_{2}, y, \omega\right)\right|^{2} \Pi(d y)=0
$$

4. the function $q_{2}(x, y, \omega)$ is continuous with $m(d y)$-probability one with respect to the argument $x$;

5. $g(0, \omega)=0$ with probability one and the Hölder condition holds:

$$
\left|g\left(x_{1}, \omega\right)-g\left(x_{2}, \omega\right)\right| \leq b \sqrt{\left|x_{1}-x_{2}\right|}
$$

with probability one for all $x_{1}, x_{2} \in \mathbb{R}$, where $b$ is a nonrandom constant;

6. $\left|a\left(x_{1}, \omega\right)-a\left(x_{2}, \omega\right)\right| \leq l\left|x_{1}-x_{2}\right|$ and

$$
\int_{\mathbb{R}}\left|q_{1}\left(x_{1}, y, \omega\right)-q_{1}\left(x_{2}, y, \omega\right)\right| \Pi(d y) \leq l\left|x_{1}-x_{2}\right|
$$

with probability one for all $x_{1}, x_{2} \in \mathbb{R}$, where $l$ is a nonrandom constant.

Then a unique strong solution of equation (2) exists.

Proof. First we prove that, for an arbitrary $T>0$, a solution of the following stochastic differential equation,

$$
X(t)=\zeta(t)+\int_{0}^{t} a(X(s)) d s+\int_{0}^{t} g(X(s)) d W(s)+\int_{0}^{t} \int_{\mathbb{R}} q_{1}(X(s), y) \tilde{\nu}(d s, d y),
$$

exists in the interval $[0, T]$.

We start with the case of $|\zeta(t)| \leq K$ for all $t \in[0, T]$ with probability one. For this, we use the theorem on uniqueness and apply the localization technique. Then we obtain a result in the general case and extend it to equation (2).

In the proof below, we apply the discretization technique known as Euler's scheme. For every $n \geq 1$, we consider a partition of the interval $[0, T]$ :

$$
0=t_{0}^{n}<t_{1}^{n}<\cdots<t_{N}^{n}=T .
$$

Denote this partition by $\Delta_{n}$. In what follows we omit the index $n$ for simplicity. The diameter of the partition is denoted by $\left\|\Delta_{n}\right\|=\sup _{1 \leq k \leq N}\left|t_{k}-t_{k-1}\right|$. In fact, we consider a sequence of partitions $\left(\Delta_{n}\right)_{n}$ with diameters approaching zero. The assumption that $\Delta_{n} \subset \Delta_{n+1}$ does not matter for our construction.

For a partition $\Delta_{n}$, we construct a stochastic process $X_{\Delta_{n}}(t)$ and denote it by $X_{n}(t)$. The process $X_{n}(t)$ is defined as follows for an argument $t$ lying between two points of the partition, that is, for $t_{k} \leq t \leq t_{k+1}, k=0, \ldots, N-1$ :

$$
\begin{aligned}
X_{n}(t)= & X_{n}\left(t_{k}\right)+\zeta(t)-\zeta\left(t_{k}\right)+a\left(X_{n}\left(t_{k}\right)\right)\left(t-t_{k}\right)+g\left(X_{n}\left(t_{k}\right)\right)\left(W(t)-W\left(t_{k}\right)\right) \\
& +\int_{\mathbb{R}} q_{1}\left(X_{n}\left(t_{k}\right), y\right)\left(\tilde{\nu}(t, d y)-\tilde{\nu}\left(t_{k}, d y\right)\right) .
\end{aligned}
$$

Here and in what follows we consider separable modifications of all stochastic processes of interest. 
Denoting $\eta_{n}(t)=t_{k}$ for $t_{k} \leq t<t_{k+1}$, we derive the following representation for the process $X_{n}(t)$ :

$$
\begin{aligned}
X_{n}(t)= & \zeta(t)+\int_{0}^{t} a\left(X_{n}\left(\eta_{n}(s)\right)\right) d s+\int_{0}^{t} g\left(X_{n}\left(\eta_{n}(s)\right)\right) d W(s) \\
& +\int_{0}^{t} \int_{\mathbb{R}} q_{1}\left(X_{n}\left(\eta_{n}(s)\right), y\right) \tilde{\nu}(d s, d y) .
\end{aligned}
$$

Following the method of mathematical induction, we show that $\mathrm{E}\left|X_{n}\left(\eta_{n}(t)\right)\right|<\infty$ for all $n \geq 1$. Indeed,

$$
\begin{aligned}
\mathrm{E}\left|X_{n}\left(t_{k+1}\right)\right|^{2} \leq 6\left(\mathrm{E}\left|X_{n}\left(t_{k}\right)\right|^{2}+2 K+\mathrm{E}\left|a\left(X_{n}\left(t_{k}\right)\right)\left(t_{k+1}-t_{k}\right)\right|^{2}\right. \\
+\mathrm{E}\left|g\left(X_{n}\left(t_{k}\right)\right)\left(W\left(t_{k+1}\right)-W\left(t_{k}\right)\right)\right|^{2} \\
\left.+\mathrm{E}\left|\int_{\mathbb{R}} q_{1}\left(X_{n}\left(t_{k}\right), y\right)\left(\tilde{\nu}\left(t_{k+1}, d y\right)-\tilde{\nu}\left(t_{k}, d y\right)\right)\right|^{2}\right) .
\end{aligned}
$$

For example, the following upper bound holds for the third term on the right-hand side of the latter inequality:

$$
\begin{aligned}
& \mathrm{E} \mid \int_{\mathbb{R}} q_{1}\left(X_{n}\left(t_{k}\right), y\right)\left(\tilde{\nu}\left(t_{k+1}, d y\right)-\left.\tilde{\nu}\left(t_{k}, d y\right)\right|^{2}\right. \\
& \quad \leq \mathrm{E} \int_{\mathbb{R}} q_{1}^{2}\left(X_{n}\left(t_{k}\right), y\right) \Pi(d y) t_{k+1}+\mathrm{E} \int_{\mathbb{R}} q_{1}^{2}\left(X_{n}\left(t_{k}\right), y\right) \Pi(d y) t_{k} \leq l\left(t_{k+1}+t_{k}\right) .
\end{aligned}
$$

Now we prove that the sequence $\mathrm{E}\left|X_{n}\left(\eta_{n}(t)\right)\right|$ is uniformly bounded with respect to $n$ and $t$. Indeed,

$$
\begin{aligned}
\mathrm{E}\left|X_{n}\left(\eta_{n}(t)\right)\right| \leq & \mathrm{E}\left|\zeta\left(\eta_{n}(t)\right)\right|+\mathrm{E}\left|\int_{0}^{\eta_{n}(t)} a\left(X_{n}\left(\eta_{n}(s)\right)\right) d s\right| \\
& +\mathrm{E}\left|\int_{0}^{\eta_{n}(t)} g\left(X_{n}\left(\eta_{n}(s)\right)\right) d W(s)\right| \\
& +\mathrm{E}\left|\int_{0}^{\eta_{n}(t)} \int_{\mathbb{R}} q_{1}\left(X_{n}\left(\eta_{n}(s)\right), y\right) \tilde{\nu}(d s, d y)\right| .
\end{aligned}
$$

The boundedness with respect to $n$ follows from

$$
\mathrm{E}\left|\int_{0}^{\eta_{n}(t)} a\left(X_{n}\left(\eta_{n}(s)\right)\right) d s\right| \leq l \int_{0}^{t}\left(1+\mathrm{E}\left|X_{n}\left(\eta_{n}(s)\right)\right|\right) d s \leq l T+l \int_{0}^{t} \mathrm{E}\left|X_{n}\left(\eta_{n}(s)\right)\right| d s
$$

Note that $|g(x)| \leq b \sqrt{|x|}$ with probability one, whence

$$
\begin{aligned}
& \mathrm{E}\left|\int_{0}^{\eta_{n}(t)} g\left(X_{n}\left(\eta_{n}(s)\right)\right) d W(s)\right| \\
& \quad \leq\left(\mathrm{E}\left|\int_{0}^{\eta_{n}(t)} g\left(X_{n}\left(\eta_{n}(s)\right)\right) d W(s)\right|^{2}\right)^{1 / 2} \leq\left(\int_{0}^{t} \mathrm{E}\left|g\left(X_{n}\left(\eta_{n}(s)\right)\right)\right|^{2} d s\right)^{1 / 2} \\
& \quad \leq\left(\int_{0}^{t} \mathrm{E} b^{2}\left|X_{n}\left(\eta_{n}(s)\right)\right| d s\right)^{1 / 2} \leq b\left(1+\int_{0}^{t} \mathrm{E}\left|X_{n}\left(\eta_{n}(s)\right)\right| d s\right)
\end{aligned}
$$


Further,

$$
\begin{aligned}
& \mathrm{E}\left|\int_{0}^{\eta_{n}(t)} \int_{\mathbb{R}} q_{1}\left(X_{n}\left(\eta_{n}(s)\right), y\right) \tilde{\nu}(d s, d y)\right| \\
& \quad \leq\left(\mathrm{E}\left|\int_{0}^{\eta_{n}(t)} \int_{\mathbb{R}} q_{1}\left(X_{n}\left(\eta_{n}(s)\right), y\right) \tilde{\nu}(d s, d y)\right|^{2}\right)^{1 / 2} \\
& \quad \leq\left(\mathrm{E} \int_{0}^{T} \int_{\mathbb{R}}\left|q_{1}\left(X_{n}\left(\eta_{n}(s)\right), y\right)\right|^{2} \Pi(d y) d s\right)^{1 / 2} \leq(l T)^{1 / 2} .
\end{aligned}
$$

Therefore

$$
\mathrm{E}\left|X_{n}\left(\eta_{n}(t)\right)\right| \leq\left(K+b+(l T)^{1 / 2}+l T\right)+(l+b) \int_{0}^{t} \mathrm{E}\left|X_{n}\left(\eta_{n}(s)\right)\right| d s .
$$

Since $\mathrm{E}\left|X_{n}\left(\eta_{n}(t)\right)\right|<\infty$, the Gronwall inequality yields

$$
\mathrm{E}\left|X_{n}\left(\eta_{n}(t)\right)\right| \leq\left(K+b+(l T)^{1 / 2}+l T\right) e^{(l+b) t}:=G_{t} \leq G_{T} .
$$

Note that the right-hand side of (5) bounds $\mathrm{E}\left|X_{n}\left(\eta_{n}(t)\right)\right|$ from above and does not depend on $n$ and on $t$. The following estimate is obtained similarly:

$$
\begin{aligned}
& \mathrm{E}\left|X_{n}(t)-X_{n}\left(\eta_{n}(t)\right)\right| \\
& \leq \mathrm{E} \mid \zeta(t)-\zeta\left(\eta_{n}(t)\right)+\int_{\eta_{n}(t)}^{t} a\left(X_{n}\left(\eta_{n}(s)\right)\right) d s \\
& \quad+\int_{\eta_{n}(t)}^{t} g\left(X_{n}\left(\eta_{n}(s)\right)\right) d W(s)+\int_{\eta_{n}(t)}^{t} \int_{\mathbb{R}} q_{1}\left(X_{n}\left(\eta_{n}(s)\right), y\right) \tilde{\nu}(d s, d y) \mid \\
& \leq l\left(1+G_{T}\right)\left\|\Delta_{n}\right\|+\mathrm{E}\left|\zeta(t)-\zeta\left(\eta_{n}(t)\right)\right|+\left(b \sqrt{G_{T}}+\sqrt{l}\right) \sqrt{\left\|\Delta_{n}\right\|} \\
& =: H_{T}(n) .
\end{aligned}
$$

Using condition 1 of Theorem 2.1 on continuity in the mean of the stochastic process $\zeta(t)$, we prove that the above majorant does not depend on $t$ and approaches zero as $n \rightarrow \infty$.

Further we prove that $\left(X_{n}\right)_{n \geq 1}$ is a Cauchy sequence in the uniform metric in the space $D[0, T]$ of real-valued right continuous functions without discontinuities of the second kind. More precisely, we prove that

$$
\mathrm{E} \sup _{0 \leq t \leq T}\left|X_{n}(t)-X_{n^{\prime}}(t)\right| \rightarrow 0
$$

as $n$ and $n^{\prime}$ increase to infinity.

We follow Yamada's method [10. Consider a sequence of functions $\varphi_{m}(u) \in \mathbf{C}^{2}(\mathbb{R})$, $m=1,2, \ldots$, approaching $|u|$. Let the numbers $1=a_{0}>a_{1}>\cdots>a_{m}>0$ be defined by the following equalities:

$$
\int_{a_{1}}^{a_{0}} \frac{d u}{b u}=1, \quad \ldots, \quad \int_{a_{m}}^{a_{m-1}} \frac{d u}{b u}=m .
$$

It is clear that $a_{m} \rightarrow 0$ as $m \rightarrow \infty$. Put

$$
\varphi_{m}(u)=\Phi_{m}(|u|),
$$


where the function $\Phi_{m}(u)$ is defined in $[0, \infty)$ and is such that $\Phi_{m} \in \mathbf{C}^{2}([0, \infty)), \Phi_{m}(0)=$ 0 , and

$$
\Phi_{m}^{\prime \prime}(u)= \begin{cases}0, & 0 \leq u \leq a_{m} \\ \text { a value between } 0 \text { and } \frac{2}{m u b}, & a_{m}<u<a_{m-1} \\ 0, & u \geq a_{m-1}\end{cases}
$$

and

$$
\int_{a_{m}}^{a_{m-1}} \Phi_{m}^{\prime \prime}(u) d u=1 .
$$

We choose $\Phi_{m}^{\prime \prime}$ to be a continuous function. Integrating $\Phi_{m}^{\prime \prime}$, we get

$$
\Phi_{m}^{\prime}(u)= \begin{cases}0, & 0 \leq u \leq a_{m} \\ \text { a value between } 0 \text { and } 1, & a_{m}<u<a_{m-1} \\ 0, & u \geq a_{m-1} .\end{cases}
$$

Now $\Phi_{m}$ is defined as the integral of $\Phi_{m}^{\prime}$.

Then

$$
\begin{aligned}
X_{n}(t)-X_{n^{\prime}}(t)= & \int_{0}^{t}\left[a\left(X_{n}\left(\eta_{n}(s)\right)\right)-a\left(X_{n^{\prime}}\left(\eta_{n^{\prime}}(s)\right)\right)\right] d s \\
& +\int_{0}^{t}\left[g\left(X_{n}\left(\eta_{n}(s)\right)\right)-g\left(X_{n^{\prime}}\left(\eta_{n^{\prime}}(s)\right)\right)\right] d W(s) \\
& +\int_{0}^{t} \int_{\mathbb{R}}\left[q_{1}\left(X_{n}\left(\eta_{n}(s)\right), y\right)-q_{1}\left(X_{n^{\prime}}\left(\eta_{n^{\prime}}(s)\right), y\right)\right] \tilde{\nu}(d s, d y) .
\end{aligned}
$$

Applying Itô's formula to the function $\varphi_{m}(x)$ we get

$$
\begin{aligned}
\varphi_{m}\left(X_{n}(t)-X_{n^{\prime}}(t)\right) \\
=\int_{0}^{t} \varphi_{m}^{\prime}\left(X_{n}(s)-X_{n^{\prime}}(s)\right)\left(a\left(X_{n}\left(\eta_{n}(s)\right)\right)-a\left(X_{n^{\prime}}\left(\eta_{n^{\prime}}(s)\right)\right)\right) d s \\
+\quad \int_{0}^{t} \varphi_{m}^{\prime}\left(X_{n}(s)-X_{n^{\prime}}(s)\right)\left(g\left(X_{n}\left(\eta_{n}(s)\right)\right)-g\left(X_{n^{\prime}}\left(\eta_{n^{\prime}}(s)\right)\right)\right) d W(s) \\
\quad+\frac{1}{2} \int_{0}^{t} \varphi_{m}^{\prime \prime}\left(X_{n}(s)-X_{n^{\prime}}(s)\right)\left(g\left(X_{n}\left(\eta_{n}(s)\right)\right)-g\left(X_{n^{\prime}}\left(\eta_{n^{\prime}}(s)\right)\right)\right)^{2} d s \\
+\int_{0}^{t} \int_{\mathbb{R}}\left[\varphi_{m}\left(X_{n}(s)-X_{n^{\prime}}(s)+q_{1}\left(X_{n}\left(\eta_{n}(s)\right), y\right)-q_{1}\left(X_{n^{\prime}}\left(\eta_{n^{\prime}}(s)\right), y\right)\right)\right. \\
\quad-\varphi_{m}\left(X_{n}(s)-X_{n^{\prime}}(s)\right) \\
\left.\quad-\left(q_{1}\left(X_{n}\left(\eta_{n}(s)\right), y\right)-q_{1}\left(X_{n^{\prime}}\left(\eta_{n^{\prime}}(s)\right), y\right)\right) \varphi_{m}^{\prime}\left(X_{n}(s)-X_{n^{\prime}}(s)\right)\right] \Pi(d y) d s \\
\quad+\int_{0}^{t} \int_{\mathbb{R}}\left[\varphi_{m}\left(X_{n}(s)-X_{n^{\prime}}(s)+q_{1}\left(X_{n}\left(\eta_{n}(s)\right), y\right)-q_{1}\left(X_{n^{\prime}}\left(\eta_{n^{\prime}}(s)\right), y\right)\right)\right] \tilde{\nu}(d s, d y) \\
=I_{1}+I_{2}+I_{3}+I_{4}+I_{5} .
\end{aligned}
$$

First we estimate $\mathrm{E}\left|I_{1}\right|$. This integral is well defined, since $\left|\varphi_{m}^{\prime}\right| \leq 1$ almost surely and $\mathrm{E}\left|X_{n}\left(\eta_{n}(u)\right)\right|$ is estimated from above by a majoarant that does not depend on $n$ 
and on $t$. Since $\left|\varphi_{m}^{\prime}\right| \leq 1$, we have

$$
\begin{aligned}
\mathrm{E} \mid \int_{0}^{t} & \varphi_{m}^{\prime}\left(a\left(X_{n}(s)\right)-a\left(X_{n^{\prime}}(s)\right)\right)\left(a\left(X_{n}\left(\eta_{n}(s)\right)\right)-a\left(X_{n^{\prime}}\left(\eta_{n^{\prime}}(s)\right)\right)\right) d s \mid \\
\leq & l \int_{0}^{t} \mathrm{E}\left|X_{n}\left(\eta_{n}(s)\right)-X_{n}(s)\right| d s+l \mathrm{E} \int_{0}^{t}\left|X_{n}(s)-X_{n^{\prime}}(s)\right| d s \\
& +l \int_{0}^{t} \mathrm{E}\left|X_{n^{\prime}}(s)-X_{n^{\prime}}\left(\eta_{n^{\prime}}(s)\right)\right| d s \\
\leq & l T H_{T}(n)+l T H_{T}\left(n^{\prime}\right)+l \mathrm{E} \int_{0}^{t}\left|X_{n}(s)-X_{n^{\prime}}(s)\right| d s .
\end{aligned}
$$

This is the bound for $\mathrm{E}\left|I_{1}\right|$ we wanted to obtain.

It is easy to see that both $I_{2}$ and $I_{5}$ are square integrable martingales and thus $\mathrm{E} I_{2}=0$ and $\mathrm{E} I_{5}=0$.

Now we estimate $\mathrm{E} I_{3}$. By the construction of the function $\varphi_{m}^{\prime \prime}$ obtained by Yamada's method, we obtain the estimate $\varphi_{m}^{\prime \prime}(u) \leq 2 /(m|u| b)$. Then

$$
\begin{aligned}
\mathrm{E} I_{3} \leq & \frac{3}{2} \mathrm{E} \int_{0}^{t} \frac{2}{m\left|X_{n}(s)-X_{n^{\prime}}(s)\right| b} b^{2}\left|X_{n}(s)-X_{n^{\prime}}(s)\right| d s \\
& +\frac{3}{2}\left\|\varphi_{m}^{\prime \prime}\right\| b^{2} \mathrm{E} \int_{0}^{t}\left(\left|X_{n}\left(\eta_{n}(s)\right)-X_{n}(s)\right|+\left|X_{n^{\prime}}(s)-X_{n^{\prime}}\left(\eta_{n^{\prime}}(s)\right)\right|\right) d s \\
\leq & \frac{3 T b}{m}+\frac{3}{2}\left\|\varphi_{m}^{\prime \prime}\right\| b^{2} T H_{T}(n)+\frac{3}{2}\left\|\varphi_{m}^{\prime \prime}\right\| b^{2} T H_{T}\left(n^{\prime}\right),
\end{aligned}
$$

where $\|\cdot\|$ denotes the uniform metric.

Finally we estimate $\mathrm{E}\left|I_{4}\right|$. Applying Lagrange's formula to the function $\varphi_{m} \in \mathbf{C}^{2}(\mathbb{R})$, we get

$I_{4}=\int_{0}^{t} \int_{\mathbb{R}}\left(\varphi_{m}^{\prime}(\theta)-\varphi_{m}^{\prime}\left(X_{n}(s)-X_{n^{\prime}}(s)\right)\right)\left(q_{1}\left(X_{n}\left(\eta_{n}(s)\right), y\right)-q_{1}\left(X_{n^{\prime}}\left(\eta_{n^{\prime}}(s)\right), y\right)\right) \Pi(d y) d s$, where $\theta=\theta(m, y)$ is a certain intermediate number. Then

$$
\begin{aligned}
\mathrm{E}\left|I_{4}\right| \leq & 2 \mathrm{E} \int_{0}^{t} \int_{\mathbb{R}}\left|q_{1}\left(X_{n}\left(\eta_{n}(s)\right), y\right)-q_{1}\left(X_{n^{\prime}}\left(\eta_{n^{\prime}}(s)\right), y\right)\right| \Pi(d y) d s \\
\leq & 2 \mathrm{E} \int_{0}^{t} l\left|X_{n}\left(\eta_{n}(s)\right)-X_{n^{\prime}}\left(\eta_{n^{\prime}}(s)\right)\right| d s \\
\leq & 2 l\left(\int_{0}^{t} \mathrm{E}\left|X_{n}\left(\eta_{n}(s)\right)-X_{n}(s)\right| d s+\int_{0}^{t} \mathrm{E}\left|X_{n}(s)-X_{n^{\prime}}(s)\right| d s\right. \\
& \left.+\int_{0}^{t} \mathrm{E}\left|X_{n^{\prime}}(s)-X_{n^{\prime}}\left(\eta_{n^{\prime}}(s)\right)\right| d s\right) \\
\leq & 2 l T\left(H_{T}(n)+H_{T}\left(n^{\prime}\right)\right)+2 l \int_{0}^{t} \mathrm{E}\left|X_{n}(s)-X_{n^{\prime}}(s)\right| d s .
\end{aligned}
$$

For a given $\varepsilon$, let $m$ be such that $0<a_{m-1}<\varepsilon / 3$ and $3 T b / m<\varepsilon / 3$. If $m$ is fixed, then $\left\|\varphi_{m}^{\prime \prime}\right\|$ is bounded. Thus there exists $n_{0}$ such that

$$
\left(H_{T}(n)+H_{T}\left(n^{\prime}\right)\right)\left(\frac{3}{2}\left\|\varphi_{m}^{\prime \prime}\right\| b^{2}+3 l\right) T<\frac{\varepsilon}{3}
$$

for all $n, n^{\prime} \geq n_{0}$ and all $t \leq T$. 
Note that $|u|-a_{m-1} \leq \varphi_{m}(u)$. Then $\left|X_{n}(t)-X_{n^{\prime}}(t)\right| \leq a_{m-1}+\varphi_{m}\left(X_{n}(t)-X_{n^{\prime}}(t)\right)$. Using the above estimates we prove that there exists $n_{0}$ such that

$$
\mathrm{E}\left|X_{n}(t)-X_{n^{\prime}}(t)\right| \leq a_{m-1}+\mathrm{E} \varphi_{m}\left(X_{n}(t)-X_{n^{\prime}}(t)\right)<\varepsilon+3 l \int_{0}^{t} \mathrm{E}\left|X_{n}(s)-X_{n^{\prime}}(s)\right| d s
$$

for all $n, n^{\prime} \geq n_{0}$ and all $t \leq T$.

The Gronwall inequality proves that $\left(X_{n}\right)_{n \geq 1}$ is a Cauchy sequence in the space $L^{1}([0, T] \times \Omega)$. Since this space is complete, we conclude that there exists a stochastic process $X$ in $L^{1}([0, T] \times \Omega)$ such that

$$
\lim _{n \rightarrow \infty} X_{n}(t, \omega)=X(t, \omega)
$$

We deduce from bound (6) that

$$
\lim _{n \rightarrow \infty} X_{n}\left(\eta_{n}(t), \omega\right)=X(t, \omega)
$$

in $L^{1}$. Hence there exists a further subsequence (for easy notation we assume without loss of generality that this subsequence coincides with the whole sequence) such that $\lim _{n \rightarrow \infty} X_{n}(t, \omega)=X(t, \omega)$ and $\lim _{n \rightarrow \infty} X_{n}\left(\eta_{n}(t), \omega\right)=X(t, \omega)$ almost everywhere with respect to the measure $d s \times d \mathrm{P}$.

To estimate $\operatorname{Esup}_{0 \leq t \leq T}\left|X_{n}(t)-X_{n^{\prime}}(t)\right|$, we use representation (4):

$$
\begin{aligned}
\mathrm{E} \sup _{0 \leq t \leq T} & \left|X_{n}(t)-X_{n^{\prime}}(t)\right| \\
\leq & l \int_{0}^{T} \mathrm{E}\left|X_{n}\left(\eta_{n}(s)\right)-X(s)\right| d s+l \int_{0}^{T} \mathrm{E}\left|X(s)-X_{n^{\prime}}\left(\eta_{n^{\prime}}(s)\right)\right| d s \\
& +\left(\mathrm{E} \sup _{0 \leq t \leq T}\left|\int_{0}^{t}\left[g\left(X_{n}\left(\eta_{n}(s)\right)\right)-g\left(X_{n^{\prime}}\left(\eta_{n^{\prime}}(s)\right)\right)\right] d W(s)\right|^{2}\right)^{1 / 2} \\
& +\left(\mathrm{E} \sup _{0 \leq t \leq T}\left|\int_{0}^{t} \int_{\mathbb{R}}\left[q_{1}\left(X_{n}\left(\eta_{n}(s)\right), y\right)-q_{1}\left(X_{n^{\prime}}\left(\eta_{n^{\prime}}(s)\right), y\right)\right] \tilde{\nu}(d s, d y)\right|^{2}\right)^{1 / 2} .
\end{aligned}
$$

Collecting the above results we prove that the first two terms on the right-hand side of the latter equality approach zero as $n, n^{\prime} \rightarrow \infty$. It remains to show the same property for the rest of the terms. By the martingale inequalities,

$$
\begin{aligned}
\mathrm{E} \sup _{0 \leq t \leq T}\left|\int_{0}^{t}\left[g\left(X_{n}\left(\eta_{n}(s)\right)\right)-g\left(X_{n^{\prime}}\left(\eta_{n^{\prime}}(s)\right)\right)\right] d W(s)\right|^{2} \\
\quad \leq 4 \mathrm{E} \int_{0}^{T}\left|g\left(X_{n}\left(\eta_{n}(s)\right)\right)-g\left(X_{n^{\prime}}\left(\eta_{n^{\prime}}(s)\right)\right)\right|^{2} d s \\
\quad \leq 4 b^{2} \mathrm{E} \int_{0}^{T}\left|X_{n}\left(\eta_{n}(s)\right)-X_{n^{\prime}}\left(\eta_{n^{\prime}}(s)\right)\right| d s \rightarrow 0, \quad n, n^{\prime} \rightarrow \infty .
\end{aligned}
$$

Similarly,

$$
\begin{gathered}
\mathrm{E} \sup _{0 \leq t \leq T}\left|\int_{0}^{t} \int_{\mathbb{R}}\left[q_{1}\left(X_{n}\left(\eta_{n}(s)\right)\right)-q_{1}\left(X_{n^{\prime}}\left(\eta_{n^{\prime}}(s)\right)\right)\right] \tilde{\nu}(d s, d y)\right|^{2} \\
\leq 4 \mathrm{E} \int_{0}^{T} \int_{\mathbb{R}}\left|q_{1}\left(X_{n}\left(\eta_{n}(s)\right), y\right)-q_{1}\left(X_{n^{\prime}}\left(\eta_{n^{\prime}}(s)\right), y\right)\right|^{2} \Pi(d y) d s \rightarrow 0, \\
n, n^{\prime} \rightarrow \infty,
\end{gathered}
$$

where we used conditions 2 and 3 of the theorem. 
Thus there exists a subsequence converging to a stochastic process that is equal almost surely (with respect to the measure $d s \times d \mathrm{P}$ ) to $X$ (this stochastic process will be denoted by the same letter). Hence $X_{n}(t)$ converges uniformly and almost surely on $[0, T]$ and, moreover,

$$
\lim _{n \rightarrow \infty} \mathrm{E} \sup _{0 \leq t \leq T}\left|X_{n}(t)-X(t)\right|=0 .
$$

Finally we show that

$$
X(t)=\zeta(t)+\int_{0}^{t} a(X(s)) d s+\int_{0}^{t} g(X(s)) d W(s)+\int_{0}^{t} \int_{\mathbb{R}} q_{1}(X(s), y) \tilde{\nu}(d s, d y) .
$$

Indeed,

$$
\begin{aligned}
\mathrm{E} \sup _{0 \leq t \leq T} \mid X(t)-\zeta(t)-\int_{0}^{t} a(X(s)) d s \\
-\int_{0}^{t} g(X(s)) d W(s)-\int_{0}^{t} \int_{\mathbb{R}} q_{1}(X(s), y) \tilde{\nu}(d s, d y) \mid \\
=\mathrm{E} \sup _{0 \leq t \leq T} \mid X(t)-X_{n}(t)+\int_{0}^{t}\left(a\left(X_{n}\left(\eta_{n}(s)\right)\right)-a(X(s))\right) d s \\
\quad+\int_{0}^{t}\left(g\left(X_{n}\left(\eta_{n}(s)\right)\right)-g(X(s))\right) d W(s) \\
\quad+\int_{0}^{t} \int_{\mathbb{R}}\left(q_{1}\left(X_{n}\left(\eta_{n}(s)\right), y\right)-q_{1}(X(s), y)\right) \tilde{\nu}(d s, d y) \mid .
\end{aligned}
$$

The further proof combines the triangle inequality and the above bounds. For example,

$$
\begin{aligned}
\mathrm{E} \sup _{0 \leq t \leq T}\left|\int_{0}^{t}\left(g\left(X_{n}\left(\eta_{n}(s)\right)\right)-g(X(s))\right) d W(s)\right| \\
\quad \leq\left(\mathrm{E} \sup _{0 \leq t \leq T}\left|\int_{0}^{t}\left(g\left(X_{n}\left(\eta_{n}(s)\right)\right)-g(X(s))\right) d W(s)\right|^{2}\right)^{1 / 2} \\
\quad \leq\left(4 \mathrm{E} \int_{0}^{T}\left|g\left(X_{n}\left(\eta_{n}(s)\right)\right)-g(X(s))\right|^{2} d s\right)^{1 / 2} \\
\quad \leq\left(4 \int_{0}^{T} \mathrm{E}\left|X_{n}\left(\eta_{n}(s)\right)-X(s)\right| d s\right)^{1 / 2} \rightarrow 0, \quad n \rightarrow \infty .
\end{aligned}
$$

Therefore we proved the existence of a solution of the stochastic differential equation (3) in the interval $[0, T]$ provided $|\zeta(t)| \leq K$ with probability one for all $t \in[0, T]$. Now we consider the general case. We introduce the sequence $\left(\sigma_{n}\right)_{n \geq 1}$ as follows:

$$
\sigma_{n}=\inf \{t:|\zeta(t)| \geq n\} \wedge T
$$

(as usual, we agree that $\inf \{\varnothing\}=T$ ). Let $\zeta^{(n)}(s):=\zeta(s) \mathbb{I}_{\left\{s<\sigma_{n}\right\}}$. Since $\left|\zeta^{(n)}(t)\right| \leq n$ for all $t \in[0, T]$, the stochastic differential equation

$$
\begin{aligned}
X^{(n)}(t)= & \zeta^{(n)}(t)+\int_{0}^{t} a\left(X^{(n)}(s)\right) d s \\
& +\int_{0}^{t} g\left(X^{(n)}(s)\right) d W(s)+\int_{0}^{t} \int_{\mathbb{R}} q_{1}\left(X^{(n)}(s), y\right) \tilde{\nu}(d s, d y)
\end{aligned}
$$

possesses a unique solution in the interval $\left[0, \sigma_{n}\right.$ [ (the proof of the uniqueness is similar to that in [5] and [7]). By the uniqueness of a solution of the stochastic differential equation (3) in $\left[0, \sigma_{n}\left[\right.\right.$, all $X^{(k)}(t), k \geq n$, are the same. Since $\bigcup_{n \geq 1}\left[0, \sigma_{n}[=[0, T[\right.$, 
and since a solution can uniquely be extended from the interval $[0, T$ to the point $T$, equation (3) possesses a unique solution on $[0, T]$.

Therefore we proved that a strong solution of equation (3) exists and is unique. The proof of the existence and uniqueness of a solution of equation (2) follows the lines of the proof of the main result in 7 .

\section{Nonnegativity of a solution of a stochastic Differential equation}

We study a generalization of the stochastic Cox-Ingersoll-Ross model in view of a practical application of stochastic differential equations for modeling instant interest

rates in financial markets. We consider the following particular case of the stochastic differential equation (2):

$$
\begin{aligned}
X(t)=\zeta( & t)+\int_{0}^{t} 2 \beta X(s) d s+\int_{0}^{t} g(X(s)) d W(s) \\
& +\int_{0}^{t} \int_{\mathbb{R}} q_{1}(X(s), y) \tilde{\nu}(d s, d y)+\int_{0}^{t} \int_{\mathbb{R}} q_{2}(X(s), y) \mu(d s, d y) .
\end{aligned}
$$

We show that a solution of this equation exists, is unique, and is nonnegative with probability one.

Theorem 3.1. We assume that the parameters of equation (8) are such that

1. $\beta \leq 0$ and $\zeta: \Omega \times \mathbb{R}^{+} \rightarrow \mathbb{R}^{+}$is an increasing stochastic process with probability one with

$$
\lim _{t_{1} \rightarrow t_{2}} \mathrm{E}\left|\zeta\left(t_{1}\right)-\zeta\left(t_{2}\right)\right|=0
$$

2. with probability one,

$$
\int_{\mathbb{R}}\left|q_{1}(x, y, \omega)\right|^{2} \Pi(d y) \leq l
$$

for all $x \in \mathbb{R}$, where $l$ is a nonrandom constant;

3. with probability one,

$$
\lim _{x_{1} \rightarrow x_{2}} \int_{\mathbb{R}}\left|q_{1}\left(x_{1}, y, \omega\right)-q_{1}\left(x_{2}, y, \omega\right)\right|^{2} \Pi(d y)=0 ;
$$

4. the function $q_{2}(x, y, \omega)$ is continuous with $m(d y)$-probability one with respect to the argument $x$ and $q_{2}(x, y, \omega) \geq 0$ with probability one for all $x, y \in \mathbb{R}$;

5. $g(0, \omega)=0$ with probability one and the following Hölder condition holds:

$$
\left|g\left(x_{1}, \omega\right)-g\left(x_{2}, \omega\right)\right| \leq b \sqrt{\left|x_{1}-x_{2}\right|}
$$

with probability one for all $x_{1}, x_{2} \in \mathbb{R}$, where $b$ is a nonrandom constant;

6. $q_{1}(0, y, \omega)=0$ with probability one for all $y \in \mathbb{R}, q_{1}(x, y, \omega) \geq 0$ with probability one for all $x \in \mathbb{R}^{+}$and $y \in \mathbb{R}$; moreover,

$$
\int_{\mathbb{R}}\left|q_{1}\left(x_{1}, y, \omega\right)-q_{1}\left(x_{2}, y, \omega\right)\right| \Pi(d y) \leq l\left|x_{1}-x_{2}\right|
$$

with probability one for all $x_{1}, x_{2} \in \mathbb{R}$, where $l$ is a nonrandom constant.

Then there exists a unique strong solution of equation (8), and this solution is nonnegative with probability one. 
Proof. The proof of the existence and uniqueness of the strong solution is the same as that in Theorem 2.1. We prove that, given a fixed $T>0$, there exists a unique solution of the stochastic differential equation

$$
X(t)=\zeta(t)+\int_{0}^{t} 2 \beta X(s) d s+\int_{0}^{t} g(X(s)) d W(s)+\int_{0}^{t} \int_{\mathbb{R}} q_{1}(X(s), y) \tilde{\nu}(d s, d y)
$$

in the interval $[0, T]$.

First we consider the case where $\zeta(T) \leq K$ with probability one.

We will show that a solution of the stochastic differential equation (9) is nonnegative. The approximation we consider in what follows may attain negative values. Thus we use the following functions:

and

$$
\tilde{g}(x)= \begin{cases}g(x), & x \geq 0, \\ 0, & x<0,\end{cases}
$$

$$
\tilde{q}_{1}(x, y)= \begin{cases}q_{1}(x, y), & x \geq 0, \\ 0, & x<0 .\end{cases}
$$

Note that the functions $\tilde{g}(x)$ and $\tilde{q}_{1}(x, y)$ satisfy the corresponding conditions of the theorem imposed on the coefficients of the stochastic differential equation.

Using the Euler scheme, we follow the lines of the proof of Theorem 2.1 to show the existence of a stochastic process $X$ in $L^{1}([0, T] \times \Omega)$ such that

$$
X(t)=\zeta(t)+\int_{0}^{t} 2 \beta X(s) d s+\int_{0}^{t} \tilde{g}(X(s)) d W(s)+\int_{0}^{t} \int_{\mathbb{R}} \tilde{q}_{1}(X(s), y) \tilde{\nu}(d s, d y) .
$$

Since $\int_{\mathbb{R}}\left|\tilde{q}_{1}(x, y)\right|^{2} \Pi(d y)$ is bounded,

$$
\int_{\mathbb{R}}\left|\tilde{q}_{1}(x, y)\right| \Pi(d y) \leq l|x|
$$

for all $x \in \mathbb{R}$ and $X \in L^{1}([0, T] \times \Omega)$, the following representation holds:

$$
\int_{\mathbb{R}} \tilde{q}_{1}(X(s), y) \tilde{\nu}(d s, d y)=\int_{\mathbb{R}} \tilde{q}_{1}(X(s), y) \nu(d s, d y)-\int_{\mathbb{R}} \tilde{q}_{1}(X(s), y) \Pi(d y) d s .
$$

Thus

$$
\begin{aligned}
X(t)= & \zeta(t)+\int_{0}^{t}\left[2 \beta X(s)-\int_{\mathbb{R}} \tilde{q}_{1}(X(s), y) \Pi(d y)\right] d s \\
& +\int_{0}^{t} \tilde{g}(X(s)) d W(s)+\int_{0}^{t} \int_{\mathbb{R}} \tilde{q}_{1}(X(s), y) \nu(d s, d y) .
\end{aligned}
$$

Next we prove that $X(t)$ is a nonnegative stochastic process. We introduce the following stopping times:

$$
\begin{gathered}
\tau_{1}=\inf \{t: X(t)<-\varepsilon\} \wedge T, \\
\sigma_{1}=\sigma_{1}^{1} \wedge \sigma_{1}^{2} \wedge T,
\end{gathered}
$$

where $\sigma_{1}^{1}=\inf \{t: X(t)<-2 \varepsilon\}$ and $\sigma_{1}^{2}=\inf \left\{t>\tau_{1}: X(t) \geq 0\right\}$.

By construction, the function $\tilde{q}_{1}(x, y)$ is nonnegative. Thus the integral with respect to the noncentered Poisson measure in (10) may lead only to positive jumps of the stochastic process $X(t)$. In other words, the stochastic process $X(t)$ has only positive jumps starting with a nonnegative point but it may also decrease eventually in a continuous way. This means, in particular, that $\tau_{1}<\sigma_{1} \leq T$.

Define the random event $A=\left\{\omega \in \Omega: \inf _{s \leq T} X(s)<-2 \varepsilon\right\}$. For almost all $\omega \in A$, we have $\sigma_{1}^{2}<\sigma_{1}^{1} \leq T$. Indeed, assuming the converse $\sigma_{1}^{1}<\sigma_{1}^{2}$, we obtain $\sigma_{1}=\sigma_{1}^{1}$, whence $X\left(\sigma_{1}\right)-X\left(\tau_{1}\right)=-\varepsilon<0$. The proof of the latter result uses a reasoning similar to the 
above one; namely, if the process $X(t)$ decreases, then it may reach the levels $-\varepsilon$ and $-2 \varepsilon$ in a continuous way and therefore $X\left(\sigma_{1}\right)=-2 \varepsilon$ and $X\left(\tau_{1}\right)=-\varepsilon$.

On the other hand,

$$
X\left(\sigma_{1}\right)-X\left(\tau_{1}\right)=\zeta\left(\sigma_{1}\right)-\zeta\left(\tau_{1}\right)+\int_{\tau_{1}}^{\sigma_{1}} 2 \beta X(s) d s \geq 0,
$$

where we used the monotonicity of the stochastic process $\zeta(t)$ in the latter inequality. Hence $X\left(\sigma_{1}\right) \geq 0$ on the random event $A$.

Further we introduce the following stopping times:

$$
\begin{gathered}
\tau_{2}=\inf \left\{t>\sigma_{1}: X(t)<-\varepsilon\right\} \wedge T, \\
\sigma_{2}=\sigma_{2}^{1} \wedge \sigma_{2}^{2} \wedge T,
\end{gathered}
$$

where $\sigma_{2}^{1}=\inf \{t \mid X(t)<-2 \varepsilon\}$ and $\sigma_{2}^{2}=\inf \left\{t>\tau_{2} \mid X(t) \geq 0\right\}$. Reasoning as above we show that $X\left(\sigma_{2}\right) \geq 0$ and $\tau_{1}<\sigma_{1}<\tau_{2}<\sigma_{2} \leq T$ on the random event $A$. Then we continue the reasoning and prove that, on the event $A$, there exists an increasing sequence of stopping times $\tau_{1}<\sigma_{1}<\cdots<\tau_{n}<\sigma_{n}<\cdots \leq T$.

Since all the stopping times do not exceed $T$, the latter sequence converges to a certain limit $\mu$. Thus $\left(\tau_{n}\right)_{n} \uparrow \mu$ and $\left(\sigma_{n}\right)_{n} \uparrow \mu$. Then, the nonnegativity of $\tilde{q}_{1}(x, y)$ for all $n$ implies that $X\left(\tau_{n}\right)=-\varepsilon$ and $X\left(\sigma_{n}\right) \geq 0$ on $A$. Hence $\mathrm{P}[A]=0$, that is, $\mathrm{P}\left[\inf _{s \leq T} X(s)<-2 \varepsilon\right]=0$. Since this property holds for all $\varepsilon>0$, the latter result means that $\mathrm{P}\left[\inf _{s \leq T} X(s)<0\right]=0$.

Since the stochastic process $X(t)$ is nonnegative, we may change $\tilde{g}$ and $\tilde{q}_{1}(x, y)$ for $g$ and $q_{1}$, respectively:

$$
\begin{aligned}
X(t)= & \zeta(t)+\int_{0}^{t} 2 \beta X(s) d s \\
& +\int_{0}^{t} g(X(s)) d W(s)+\int_{0}^{t} \int_{\mathbb{R}} q_{1}(X(s), y) \tilde{\nu}(d s, d y) .
\end{aligned}
$$

This completes the proof of the existence of a nonnegative solution of the stochastic differential equation (8) in the interval $[0, T]$ provided that $\zeta(T) \leq K$ with probability one. The proof of the existence and uniqueness of a solution of the stochastic differential equation (8) for the general case is the same as that in Theorem 2.1. This solution is nonnegative, since the function $q_{2}(x, y)$ is nonnegative.

Remark 3.1. Theorem 3.1 holds for a drift coefficient $a(x, \omega): \mathbb{R} \times \Omega \rightarrow \mathbb{R}^{+}$that satisfies the conditions of Theorem 2.1 (this follows from the proof of the latter theorem, since the integral in equality (11) is nonnegative if the drift coefficient is nonnegative).

Lemma 3.1. Theorem 3.1 holds for the stochastic differential equation with a diffusion $g(x)$ such that $|g(x)|=b x^{\alpha}, \frac{1}{2} \leq \alpha<1$.

Proof. Consider the stochastic differential equation

$$
\begin{aligned}
X^{(h)}(t)= & \zeta(t)+\int_{0}^{t} 2 \beta X^{(h)}(s) d s \\
& +\int_{0}^{t} g_{h}\left(X^{(h)}(s)\right) d W(s)+\int_{0}^{t} \int_{\mathbb{R}} q_{1}\left(X^{(h)}(s), y\right) \tilde{\nu}(d s, d y),
\end{aligned}
$$

where $g_{h}(x)=b x^{\alpha}$ for $x \leq h$ and $g_{h}(x)=b h^{\alpha}$ for $x \geq h$. The function $g_{h}$ satisfies the Hölder condition:

$$
\left|g_{h}\left(x_{1}\right)-g_{h}\left(x_{2}\right)\right| \leq b_{h} \sqrt{\left|x_{1}-x_{2}\right|} .
$$

Thus Theorem 3.1 implies that a solution of stochastic differential equation (12) exists in the interval $\left[0, \sigma_{h}\right]$ and is unique and nonnegative, where the sequence $\left(\sigma_{h}\right)_{h \geq 1}$ is such 
that $\sigma_{h}=\inf \left\{t \mid X^{(h)}(t) \geq h\right\}$. In the interval $\left[0, \sigma_{h}\right]$, all the solutions $X^{(k)}, k \geq h$, coincide in view of the uniqueness.

We show that $\mathrm{P}\left[\sup _{0 \leq t \leq T} X^{(h)}(t) \geq h\right] \rightarrow 0$ as $h \rightarrow \infty$. The Chebyshev inequality implies that

$$
\mathrm{P}\left[\sup _{0 \leq t \leq T} X^{(h)}(t) \geq h\right] \leq \frac{1}{h} \mathrm{E} \sup _{0 \leq t \leq T} X^{(h)}(t) .
$$

Since the process $X^{(h)}$ is nonnegative, the bounds obtained in the proof of Theorem 3.1 yield

$$
\begin{aligned}
\mathrm{E} \sup _{0 \leq t \leq T} X^{(h)}(t)= & \mathrm{E} \sup _{0 \leq t \leq T}\left(\zeta(t)+\int_{0}^{t} 2 \beta X^{(h)}(s) d s\right. \\
& \left.\quad+\int_{0}^{t} g_{h}\left(X^{(h)}(s)\right) d W(s)+\int_{0}^{t} \int_{\mathbb{R}} q_{1}\left(X^{(h)}(s), y\right) \tilde{\nu}(d s, d y)\right) \\
\leq & \mathrm{E} \zeta(T)+\left(4 \mathrm{E} \int_{0}^{T} g_{h}^{2}\left(X^{(h)}(s)\right) d s\right)^{1 / 2} \\
& +\left(4 \mathrm{E} \int_{0}^{T} q_{1}^{2}\left(X^{(h)}(s), y\right) \Pi(d y) d s\right)^{1 / 2} \\
\leq & \mathrm{E} \zeta(T)+\left(4 T b^{2} h^{2 \alpha}\right)^{1 / 2}+\left(4 T l^{2}\right)^{1 / 2}=\mathrm{E} \zeta(t)+2 T^{1 / 2}\left(b h^{\alpha}+l\right)
\end{aligned}
$$

whence we obtain that

$$
\mathrm{P}\left[\sup _{0 \leq t \leq T} X^{(h)}(t) \geq h\right] \rightarrow 0 \quad \text { as } h \rightarrow \infty
$$

and thus $\bigcup\left[0, \sigma_{h}\right]=[0, \infty)$.

This completes the proof of the existence of a unique strong solution on $\mathbb{R}^{+} \times \Omega$ for the stochastic differential equation (9) with a diffusion $g(x)$ such that $|g(x)|=b x^{\alpha}$, $\frac{1}{2} \leq \alpha<1$, and with a centered Poisson measure. The extension of this result to the case of equation (8) can be done in the same way as in the proof of Theorem 3.1

\section{A BOUND FOR THE PROBABILITY THAT A SOLUTION OF A STOCHASTIC DIFFERENTIAL EQUATION ASSUMES A NEGATIVE VALUE}

Estimates for the probability that a solution of the stochastic differential equation attains a negative value are of practical interest when evaluating the ruin probability of an insurance company. In the classical Lundberg collective risk model, claims are modeled with the help of a homogeneous Poisson process. Many papers are devoted to generalizations of this model. In particular, the paper [11] deals with a model of an insurance company which invests a part of its reserve capital to risky assets, while the rest is invested to nonrisky assets. The price of risky assets is modeled with the help of a geometric Brownian motion, while the claims constitute a compound Poisson process. An exponential bound is obtained in [11] for the ruin probability.

Consider the following stochastic differential equation:

$$
X_{u}(t)=u+\int_{0}^{t} a\left(X_{u}(s)\right) d s+\int_{0}^{t} g\left(X_{u}(s)\right) d W(s)+\int_{0}^{t} \int_{\mathbb{R}} q_{1}\left(X_{u}(s), y\right) \tilde{\nu}(d s, d y)
$$

$u>0$. We estimate from above the probability that a solution $X_{u}$ of equation (13) attains a negative value. Put $\tau(u):=\inf \left\{t \geq 0: X_{u}(t) \leq 0\right\}$; if $X_{u}(t)>0$ for all $t>0$, then we put $\tau(u)=+\infty$. 
Theorem 4.1. Assume that the coefficients of equation (13) are such that

1. with probability one,

$$
|a(x, \omega)| \leq l(1+|x|), \quad \int_{\mathbb{R}}\left|q_{1}(x, y, \omega)\right|^{2} \Pi(d y) \leq l
$$

for all $x \in \mathbb{R}$, where $l$ is a nonrandom constant;

2. with probability one,

$$
\lim _{x_{1} \rightarrow x_{2}} \int_{\mathbb{R}}\left|q_{1}\left(x_{1}, y, \omega\right)-q_{1}\left(x_{2}, y, \omega\right)\right|^{2} \Pi(d y)=0 ;
$$

3. $g(0, \omega)=0$ with probability one and the following Hölder condition holds:

$$
\left|g\left(x_{1}, \omega\right)-g\left(x_{2}, \omega\right)\right| \leq b \sqrt{\left|x_{1}-x_{2}\right|}
$$

with probability one for all $x_{1}, x_{2} \in \mathbb{R}$, where $b$ is a nonrandom constant;

4. with probability one,

$$
\left|a\left(x_{1}, \omega\right)-a\left(x_{2}, \omega\right)\right| \leq l\left|x_{1}-x_{2}\right|
$$

for all $x_{1}, x_{2} \in \mathbb{R}$, where $l$ is a nonrandom constant;

5. $q_{1}(x, y, \omega) \geq 0$ with probability one for all $x \in \mathbb{R}^{+}$and $y \in \mathbb{R}$; moreover,

$$
\int_{\mathbb{R}}\left|q_{1}\left(x_{1}, y, \omega\right)-q_{1}\left(x_{2}, y, \omega\right)\right| \Pi(d y) \leq l\left|x_{1}-x_{2}\right|
$$

with probability one for all $x_{1}, x_{2} \in \mathbb{R}$, where $l$ is a nonrandom constant;

6. there exists a finite number $r>0$ such that

$$
\frac{1}{2} r^{2} g^{2}(x)-a(x) r+\int_{\mathbb{R}}\left[e^{-r q_{1}(x, y)}-1+r q_{1}(x, y)\right] \Pi(d y) \leq 0
$$

with probability one for all $x \geq 0$.

Then

$$
\mathrm{P}\{\tau(u)<\infty\} \leq e^{-r u} .
$$

Proof. The assumptions of the theorem guarantee that a strong solution of the stochastic differential equation (13) exists. Consider the stochastic process $Y_{u}(t, r):=e^{-r X_{u}(t)}$. We prove that $Y_{u}(t \wedge \tau(u))$ is a supermartingale. This result follows if $Y_{u}(t \wedge \tau(u))$ is integrable and if

$$
E_{t \wedge \tau(u)}\left[Y_{u}(T \wedge \tau(u), r)-Y_{u}(t \wedge \tau(u), r)\right] \leq 0
$$

holds almost surely for all $T>t \geq 0$. The symbol $E_{t}(\cdot)$ stands here for the conditional mathematical expectation $\mathrm{E}\left(\cdot \mid \mathcal{F}_{t}\right)$.

Our current goal is to find a representation for $e^{-r X(t)}$. Applying the generalized Itô formula to the function $f(x)=e^{-r x}$ we obtain

$$
\begin{aligned}
e^{-r X(t \wedge \tau)}= & e^{-r u}+\int_{0}^{t \wedge \tau} e^{-r X(s)}\left[\frac{1}{2} r^{2} g^{2}(X(s))-r a(X(s))\right] d s \\
& -\int_{0}^{t \wedge \tau} r e^{-r X(s)} g(X(s)) d W(s) \\
& +\int_{0}^{t \wedge \tau} \int_{\mathbb{R}} e^{-r X(s)}\left[e^{-r q_{1}(X(s), y)}-1+r q_{1}(X(s), y)\right] \Pi(d y) d s \\
& +\int_{0}^{t \wedge \tau} \int_{\mathbb{R}} e^{-r(X(s))}\left[e^{-r q_{1}(X(s), y)}-1\right] \tilde{\nu}(d s, d y) .
\end{aligned}
$$


Now we prove that $Y(t \wedge \tau)$ is integrable. Similarly to bound (5) we prove that $\mathrm{E}|X(t)|$ is bounded. Considering representation (14) we get

$$
\begin{aligned}
\mathrm{E} e^{-r X(t \wedge \tau)} \leq & e^{-r u}+\mathrm{E}\left|\int_{0}^{t \wedge \tau} e^{-r X(s)}\left[\frac{1}{2} r^{2} g^{2}(X(s))-r a(X(s))\right] d s\right| \\
& +\mathrm{E}\left|\int_{0}^{t \wedge \tau} \int_{\mathbb{R}} e^{-r X(s)}\left[e^{-r q_{1}(X(s), y)}-1+r q_{1}(X(s), y)\right] \Pi(d y) d s\right|<\infty .
\end{aligned}
$$

Indeed,

$$
\mathrm{E}\left|\int_{0}^{t \wedge \tau} e^{-r X(s)} g^{2}(X(s)) d s\right| \leq \int_{0}^{t \wedge \tau} b \mathrm{E}|X(s)| d s<\infty .
$$

Then we use the inequality $e^{x}-1-x \leq x^{2} / 2, x \leq 0$, and obtain

$$
\begin{gathered}
\mathrm{E}\left|\int_{0}^{t \wedge \tau} \int_{\mathbb{R}} e^{-r X(s)}\left[e^{-r q_{1}(X(s), y)}-1+r q_{1}(X(s), y)\right] \Pi(d y) d s\right| \\
\leq \frac{1}{2} \mathrm{E} \int_{0}^{t \wedge \tau} \int_{\mathbb{R}} e^{-r X(s)} q_{1}^{2}(X(s), y) \Pi(d y) d s<\infty
\end{gathered}
$$

in view of the boundedness of $\int_{\mathbb{R}}\left|q_{1}(x, y)\right|^{2} \Pi(d y)$ and according to the definition of the random variable $\tau(u)$. Therefore, the process $Y(t \wedge \tau)$ is bounded.

Further,

$$
\begin{aligned}
& E_{t \wedge \tau}[Y(T \wedge \tau)-Y(t \wedge \tau)] \\
& =E_{t \wedge \tau}\left(\int_{t \wedge \tau}^{T \wedge \tau} e^{-r X(s)}\left[\frac{1}{2} r^{2} g^{2}(X(s))-r a(X(s))\right] d s\right. \\
& -\int_{t \wedge \tau}^{T \wedge \tau} r e^{-r X(s)} g(X(s)) d W(s) \\
& +\int_{t \wedge \tau}^{T \wedge \tau} \int_{\mathbb{R}} e^{-r(X(s))}\left[e^{-r q_{1}(X(s), y)}-1+r q_{1}(X(s), y)\right] \Pi(d y) d s \\
& \left.+\int_{t \wedge \tau}^{T \wedge \tau} \int_{\mathbb{R}} e^{-r X(s)}\left[e^{-r q_{1}(X(s), y)}-1\right] \tilde{\nu}(d s, d y)\right) \\
& \leq \int_{t \wedge \tau}^{T \wedge \tau} e^{-r X(s)}\left(\frac{1}{2} r^{2} g^{2}(X(s))-r a(X(s))\right. \\
& \left.+\int_{\mathbb{R}}\left[e^{-r q_{1}(X(s), y)}-1+r q_{1}(X(s), y)\right] \Pi(d y)\right) d s
\end{aligned}
$$

$\leq 0$.

The latter inequality follows from condition 6 of the theorem. Inequality (17) holds, since both terms (15) and (16) are martingales.

Thus $Y_{u}(t \wedge \tau(u))$ is a supermartingale. Then

$$
\begin{aligned}
e^{-r u} & =Y_{u}(0, r) \geq \mathrm{E} Y_{u}(t \wedge \tau(u), r) \\
& =\mathrm{E} Y_{u}(\tau(u), r) \cdot I_{\{\tau(u)<t\}}+\mathrm{E} Y_{u}(t, r) \cdot I_{\{\tau(u) \geq t\}} \geq \mathrm{E} Y_{u}(\tau(u), r) \cdot I_{\{\tau(u)<t\}} .
\end{aligned}
$$

Since

$$
\lim _{t \rightarrow \infty} \mathrm{E} Y_{u}(\tau(u), r) \cdot I_{\{\tau(u)<t\}}=\mathrm{E} Y_{u}(\tau(u), r) \cdot I_{\{\tau(u)<\infty\}},
$$

we have

$$
e^{-r u} \geq \mathrm{E}\left(Y_{u}(\tau(u), r) / \tau(u)<\infty\right) \cdot \mathrm{P}\{\tau(u)<\infty\},
$$

whence

$$
\mathrm{P}\{\tau(u)<\infty\} \leq \frac{e^{-r u}}{\mathrm{E} Y_{u}(\tau(u), r)} \leq e^{-r u} .
$$


The latter inequality holds in view of the following two bounds: $X_{u}(\tau(u), r) \leq 0$ and $Y_{u}(\tau(u), r) \geq 1$.

\section{Concluding Remarks}

Studies of properties of solutions of stochastic differential equations with random coefficients, non-Lipschitzian diffusion, and with a jump part are of special interest from the point of view of applications. Equations of this type can be used to model the interest rates that may jump eventually in financial markets.

We proved the existence and uniqueness of a strong solution of the stochastic differential equation with random coefficients and non-Lipschitzian diffusion for both cases, centered and noncentered Poisson measure. We provide conditions that a solution of an equation of such a type is nonnegative and estimate from above the probability that a solution attains a negative value.

\section{BIBLIOGRAPHY}

1. J. C. Cox, J. E. Ingersoll, and S. A. Ross, A theory of the term structure of interest rates, Econometrica 53 (1985), 385-407. MR785475

2. V. Zubchenko, Long-term returns in stochastic interest rate models, Theory Stoch. Process. 13(29) (2007), no. 4, 247-261. MR2482264 (2010h:60188)

3. I. I. Gikhman and A. V. Skorokhod, Stochastic Differential Equations and their Applications, Naukova dumka, Kiev, 1982. (Russian) MR678374 (84j:60003)

4. N. Ikeda and S. Watanabe, Stochastic Differential Equations and Diffusion Processes, NorthHolland Publishing Co./Kodansha, Ltd., Amsterdam-New York/Tokyo, 1981. MR637061 (84b:60080)

5. G. L. Kulinich, On existence and uniqueness of a solution of a stochastic differential equation with martingale differential, Teor. Veroyatnost. i Primenen. XIX (1974), no. 1, 169-173; English transl. in Theory Probab. Appl. 19 (1975), 168-171. MR0345209 (49:9948)

6. G. Deelstra and F. Delbaen, Convergence of discretized stochastic (interest rate) processes with stochastic drift term, Appl. Stochastic Models Data Anal. 14 (1998), 77-84. MR1641781 (99g:60097)

7. V. P. Zubchenko and Yu. S. Mishura, Existence and uniqueness of solutions of stochastic differential equations with non-Lipschitz diffusion and Poisson measure, Teor. Imovir. Mat. Stat. 80 (2009), 43-54; English transl. in Theory Probab. Math. Statist. 80 (2010), 47-59. MR2541951 (2010h:60189)

8. I. I. Gikhman and A. V. Skorokhod, Stochastic Differential Equations, Naukova dumka, Kiev, 1968; English transl., Springer-Verlag, New York-Heidelberg, 1972. MR0263172 (41:7777) MR0346904 (49:11625)

9. R. J. Elliott, Stochastic Calculus and Applications, Springer-Verlag, New York-HeidelbergBerlin, 1986. MR678919 (85b:60059)

10. T. Yamada, Sur une construction des solutions d'équations différentielles stochastiques dans le cas non-lipshitzien, Sém. de Prob. XII (1978), Lecture Notes in Math. 649, 114-131. MR.520000 (80k:60076)

11. J. Gaier, P. Grandits, and W. Schachermayer, Asymptotic ruin probabilities and optimal investment, Ann. Appl. Probab. 13 (2003), 1054-1076. MR1994044 (2004k:91124)

Department of Probability Theory, Statistics, and Actuarial Mathematics, Faculty for Mechanics and Mathematics, National Taras Shevchenko University, Academician Glushkov Avenue 2, Kiev 03127, Ukraine

E-mail address: v_zubchenko@ukr.net

Received 10/JUL/2009

Translated by N. SEMENOV 